\title{
High-Resolution 3T MR Neurography of the Brachial Plexus and Its Branches, with Emphasis on 3D Imaging
}

\author{
A. Chhabra, G.K. Thawait, T. Soldatos, R.S. Thakkar, F. Del Grande, M. Chalian, and J.A. Carrino
}

\begin{abstract}
SUMMARY: With advancement in 3D imaging, better fat-suppression techniques, and superior coil designs for MR imaging and the increasing availability and use of 3T magnets, the visualization of the complexity of the brachial plexus has become facile. The relevant imaging findings are described for normal and pathologic conditions of the brachial plexus. These radiologic findings are supported by clinical and/or EMG/surgical data, and corresponding high-resolution MR neurography images are illustrated. Because the brachial plexus can be affected by a plethora of pathologies, resulting in often serious and disabling complications, a better radiologic insight has great potential in aiding physicians in rendering superior services to patients.
\end{abstract}

ABBREVIATIONS: $E M G$ = electromyography; MIP = maximum intensity projection; MRN = MR neurography; SPACE = sampling perfection with applicationoptimized contrasts by using different flip angle; STIR = short tau inversion recovery, TOS = thoracic outlet syndrome

$\mathbf{T}$ he brachial plexus is a network of nerve confluences and ramifications, which combine to form the large terminal branches that supply motor and sensory branches to the upper extremities. The clinical differentiation of brachial plexopathy from other spine-related abnormalities often poses a considerable diagnostic challenge, and electrodiagnostic tests are difficult to perform due to the deep location of the plexus, often leading to indeterminate results. MRN has been increasingly used in recent years to further evaluate cases of suspected or established brachial plexopathy and peripheral neuropathy, with excellent results. ${ }^{1-4}$ This article illustrates the relevant anatomy and the various common pathologies of the brachial plexus and describes the respective imaging findings at $3 \mathrm{~T}$ MR neurography, with emphasis on 3D imaging.

\section{ANATOMIC CONSIDERATIONS}

\section{Nerve Roots and Their Anatomic Relations}

The ventral rami of the C5-C8 and T1 nerve roots unite to form the brachial plexus, between the anterior and middle scalene muscles. It may receive additional contributions from the $\mathrm{C} 4$ and $\mathrm{T} 2$ nerve roots. The dorsal scapular nerve (C5) and long thoracic

From the Russell H. Morgan Department of Radiology and Radiological Science, Johns Hopkins Hospital, Baltimore, Maryland.

The authors report no conflicts of interest.

Please address correspondence to Avneesh Chhabra, MD, Assistant Professor Radiology \& Orthopedic Surgery, Russell H. Morgan Department of Radiology \& Radiological Science, The Johns Hopkins Hospital, 601 N. Caroline St, JHOC 3262, Baltimore, MD 21287; e-mail: achhabr6@jhmi.edu

- Indicates open access to non-subscribers at www.ajnr.org

http://dx.doi.org/10.3174/ajnr.A3287 nerve (C5-C7) to the serratus anterior muscle arise directly from the nerve roots. ${ }^{1,2,5}$

\section{Trunks and Their Anatomic Relations}

The C5 and C6 nerve roots compose the upper trunk, C7 continues as the middle trunk, and C8 and T1 compose the lower trunk. The 3 trunks are formed within the interscalene triangle. The suprascapular nerve (C5 and C6) and the nerve to the subclavius arise from the superior trunk. The phrenic nerve (C3-C5) passes between the anterior and middle scalene and continues over the surface of the anterior scalene muscle.

\section{Divisions, Cords, and Their Anatomic Relations}

More laterally, the trunks ramify into the 3 anterior and 3 posterior divisions, which join to form 3 cords distal to the lateral margin of the first rib. The cords are the lateral, posterior, and medial cords, based on their relationship to the axillary artery. The lateral cord is formed by the anterior divisions of the upper and middle trunks and gives off the lateral pectoral nerve (C5-C7) and contributes to the median and the musculocutaneous nerves. The posterior divisions of all the trunks form the posterior cord, which gives off the subscapular nerves. The inferior trunk continues as the medial cord and gives off the median pectoral nerve $(\mathrm{C} 8, \mathrm{~T} 1)$, the medial brachial cutaneous nerve (T1), and the medial antebrachial cutaneous nerve $(\mathrm{C} 8, \mathrm{~T} 1)$.

\section{Branches}

The cords end in 5 terminal branches: the median, ulnar, musculocutaneous, axillary, and radial nerves. The respective 
3T MRN examination protocol for the evaluation of the brachial plexus (FOVs from C2 to T2)

\begin{tabular}{lcccc}
\hline & FOV & $\begin{array}{c}\text { Section } \\
\text { Thickness }\end{array}$ & $\begin{array}{c}\text { TR/TE/TF } \\
\text { (ms) }\end{array}$ & Matrix \\
\hline MR imaging sequence & & & & \\
T1 coronal (T1 axial) & 30 & 4.0 & $881 / 11 / 7$ & $512 \times 512$ \\
3D coronal STIR SPACE & 30 & 1.0 & $1500 / 97 / 53$ & $256 \times 256$ \\
3D sagittal T2 SPACE & 25 & 1.0 & $1000 / 97 / 81$ & $256 \times 256$ \\
$\quad$ STIR sagittal, bilateral, affected side & $22-24$ & 3.0 & $5210 / 18 / 22$ & $256 \times 256$ \\
Additional arm examination if desired & & & & \\
$\quad$ Axial T1 & $20-22$ & $3.0-4.0$ & $550 / 7.9 / 6$ & $256 \times 384$ \\
$\quad$ Axial T2 SPAIR & $20-22$ & $3.0-4.0$ & $2840 / 70 / 13$ & $256 \times 384$ \\
\hline
\end{tabular}

Note:-TF indicates turbo factor; SPAIR, spectral-attenuated inversion recovery.

anatomy and regional lesions of these nerves will be discussed in the last section of the article.

\section{PATHOLOGIC CONDITIONS}

Brachial plexus lesions can be prudently classified according to their location in relation to the clavicle, as supraclavicular, retroclavicular, and infraclavicular lesions. Less commonly, panplexus lesions may result from severe trauma or radiation neuropathy.

\section{Supraclavicular Lesions}

Supraclavicular lesions involve nerve roots and trunks in the scalene triangle, and generally the pathology is more commonly encountered and more severe than lesions at other sites. Erb-Duchenne palsy results from injury to the $\mathrm{C} 5$ and $\mathrm{C} 6$ roots or the upper trunk and accounts for approximately $90 \%$ of obstetric brachial plexus injuries. Much less common is Dejerine-Klumpke palsy, which results from injury to the C8 and T1 roots or the lower trunk. Common pathologies in the supraclavicular area include brachial plexitis (Parsonage-Turner syndrome), traumatic injury, neoplasms (metastasis, nerve sheath tumor, neurocutaneous syndrome, pancoast tumor), and TOS. C5 and C6 (upper plexus) involvement leads to an adducted and internally rotated arm with weakness in the shoulder and flexor arm muscles, as well as sensory loss in the lateral arm and thumb. C7 (middle trunk) involvement leads to weakness of arm extension and forearm pronation with sensory loss in the middle finger. C8 and T1 (lower plexus) involvement leads to weakness of intrinsic hand muscles with sensory loss in the ulnar aspect of the forearm and hand. ${ }^{5-8}$

\section{Retroclavicular Lesions}

Retroclavicular lesions involve the brachial plexus divisions. Generally, anterior division lesions lead to flexor compartment pathology, and posterior division lesions lead to extensor compartment pathology. Isolated lesions in the divisions are rare; therefore, for practical purposes, these are included in the infraclavicular pathology.

\section{Infraclavicular Lesions}

Infraclavicular lesions affect the cords and terminal branch nerves, and these are approximately 3 times less commonly seen than the supraclavicular lesions. These lesions also generally have better prognosis and earlier recovery than the supraclavicular lesions. Common causes include radiation neuropathy, humeral fracture-dislocation, gunshot injury, and iatrogenic injuries. Lateral cord lesions cause weakness in elbow/wrist flexion and fore- arm pronation with sensory loss in the anterolateral forearm and hand. Medial cord lesions produce similar symptoms with relative sparing of the finger extensors. Posterior cord lesions affect the radial and axillary nerves, causing weak arm, wrist, and finger extension and sensory loss over the shoulder and posterior arm.

\section{MRN INDICATIONS}

MRN is indicated for the following reasons: 1) in patients with nonspecific shoulder and arm pain or weakness, in which EMG and traditional MR imaging of the spine are inconclusive, to rule in or rule out brachial plexopathy; 2) to evaluate anatomy and confirm nerve morphologic abnormalities in patients under consideration for surgery for TOS; 3 ) in patients with symptoms of brachial plexopathy with a history of radiation therapy to exclude recurrent malignancy/confirm radiation plexopathy; 4) to characterize and evaluate the extent of space-occupying lesions, such as hematoma, nerve sheath tumor, metastatic disease, and so forth; 5) to evaluate and differentiate a simple stretch injury from higher grade nerve injury; 6) to evaluate clinically suspected diffuse polyneuropathy and tumorlike conditions; 7) to exclude nerve re-entrapment/persistent impingement in failed surgery cases, and 8) for guidance in perineural and scalene medication injections. ${ }^{1-12}$

\section{MRN Technique and Normal Imaging Appearances}

The MRN technique used in our institution has been outlined in the Table. Although MRN can be performed at lower field strengths, we perform most of our examinations on a 3T scanner (Trio, Verio, Skyra; Siemens, Erlangen, Germany) due to the superior SNR and contrast. 3T imaging provides high-quality $2 \mathrm{D}$ and $3 \mathrm{D}$ imaging, essential for the optimal evaluation of the complex anatomy, frequent variations, and small size of the brachial plexus segments (Fig 1). T1-weighted images are most useful for anatomy of the nerves, surrounding fat planes, scalene and regional muscles, and the thoracic outlet. T2-weighted images are obtained with a variety of contrasts (fat-suppressed T2weighted/STIR) for optimal assessment of the brachial plexus. Sagittal STIR imaging is useful for individual-segmental assessment of the nerve segments. STIR imaging has multiple advantages, such as depiction of symmetric normal hyperintensity and size characteristics of the dorsal nerve root ganglion at multiple levels on the same image for comparison. The nerve roots can be followed throughout their course from their origin to the terminal segments of the brachial plexus, and any relative change in signal and morphology among ipsilateral and contralateral respective nerve can be easily identified. Relative identification of trunks and cords is facilitated by their relationships to the subclavian and axillary arteries, respectively; and finally, the intramuscular course of the nerves through the scalene muscle is nicely depicted as a step-off in the normal smooth course.

The 3D STIR SPACE sequence allows excellent background fat suppression and isotropic multiplanar and curved planar recon- 


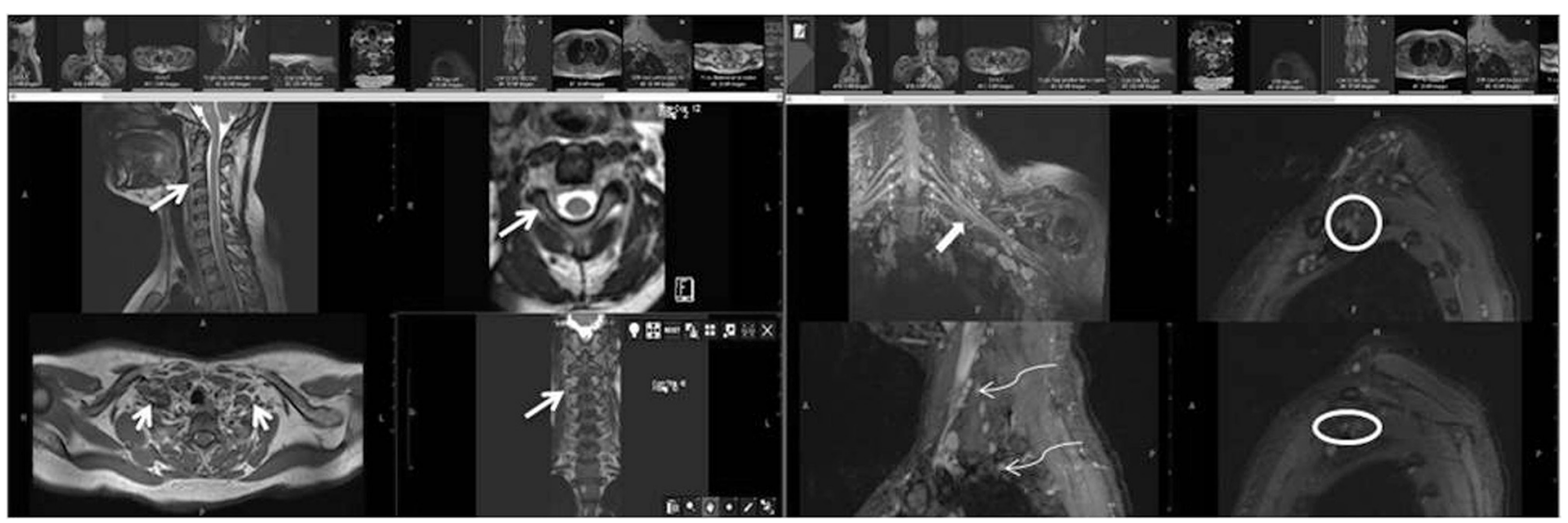

FIG 1. MRN technique. 3T MR neurography imaging sequences with isotropic multiplanar reconstruction. 3D T2 SPACE with multiplanar reconstruction of the cervical spine (medium arrows). Axial TI-weighted image shows the bilateral brachial plexus through the lower aspect of the scalene triangle (small arrows). Coronal MIP 3D STIR SPACE image shows the brachial plexus (thick arrow). Sequential sagittal STIR images show the normal and symmetric C5-T1 nerve roots (curved arrows), trunks (circle), and cords (oval).

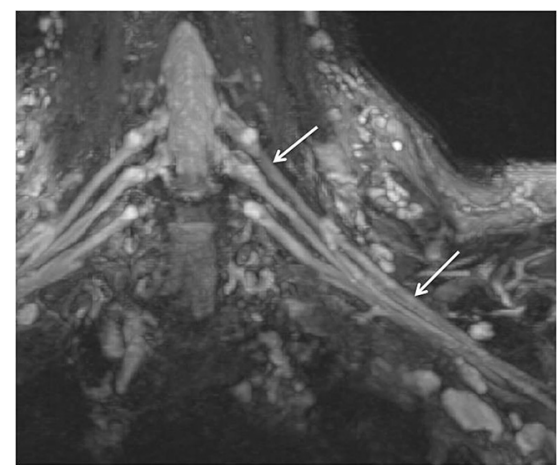

FIG 2. Normal brachial plexus. Coronal MIP 3D STIR SPACE image focused on the left side shows the anatomy of the brachial plexus (arrows).

structions. The reconstructed images can be further enhanced with MIP to highlight the imaging abnormality and for depiction along the longitudinal plane of the nerve. With current $3 \mathrm{~T}$ magnet strength and flex coils, SNR decreases near the shoulder point on high-resolution 3D images if both sides are imaged together. Therefore, if a unilateral abnormality is suspected in the distal segments (cords) of the brachial plexus, ipsilateral 3D imaging focused to that area should be performed (Fig 2). Sagittally acquired STIR images often circumvent this limitation.

Finally, 3D T2 SPACE images focused on the cervical spine are important, as spine lesions can be a major confounder in mimicking brachial plexus pathology. Additionally, pre-ganglionic intradural nerve segments are best identified on this sequence. Overhead (superman position) imaging has been described in the literature. Due to the frequent variations in thoracic outlet compromise and neurovascular compression in healthy volunteers and patients, we do not routinely perform these added imaging maneuvers, unless specifically requested by the clinicians. Although it has also been shown in the literature that enhancement of intradural nerve roots suggests their functional impairment despite apparent morphologic continuity due to a disrupted blood-nerve barrier, it is a very uncommon finding in the authors' experience. It might be related to the subacute nature of these injuries when they come for MRN imaging. Generally, all the brachial plexus studies are performed as unenhanced MRN examinations, unless there is a suspicion of neoplasm, infection, or diffuse polyneuropathy. ${ }^{3,4,11,13,14}$

DTI is experimental at this stage. It provides insight into nerve microarchitecture by reconstruction of DTI parametric maps with illustration of the fiber tracts. From the postprocessing of DTI data, a fractional anisotropy color-coded map or tractography pattern can be generated by using available DTI software. Fractional anisotropy maps exhibit the structures with high anisotropy, which, when coregistered with other anatomic sequences, can be used to localize these regions. Because the DTI protocols are being standardized and initial reliability studies are being performed, it is likely to play an important role in peripheral nerve imaging, including the brachial plexus. ${ }^{15,16}$

MRN imaging anatomy of the thoracic outlet can be subdivided into 3 anatomic spaces, from medial to lateral: the interscalene triangle, the costoclavicular space, and the retropectoralis minor space (also called the subcoracoid tunnel). These are best characterized on sagittal and coronal images. The interscalene triangle is bordered anteriorly by the anterior scalene muscle, posteriorly by both the middle and posterior scalene muscles, and inferiorly by the first rib. The interscalene triangle contains the subclavian vessels, roots, and trunks of the brachial plexus. The costoclavicular space is bounded anteriorly by the subclavius muscle, anterosuperiorly by the clavicle, and posteriorly by both the first rib and the middle scalene muscle. This space contains the subclavian vessels, and the divisions and cords of the brachial plexus. The most lateral portion of the thoracic outlet, the retropectoralis minor space (subcoracoid tunnel), is defined by the pectoralis minor anteriorly, the subscapularis muscle posteriorly and superiorly, and the anterior chest wall posteroinferiorly. It contains the distal cords and the terminal branches of the brachial plexus. The cords are adjacent to the axillary artery in the following locations (in clockwise order): anterosuperior (lateral cord), superoposterior (posterior cord), and posteroinferior (medial cord) in the axilla. The terminal branches are usually difficult to separate unless concomitant imaging of the arm is performed or if they are involved in neuropathy. ${ }^{17,18}$ 

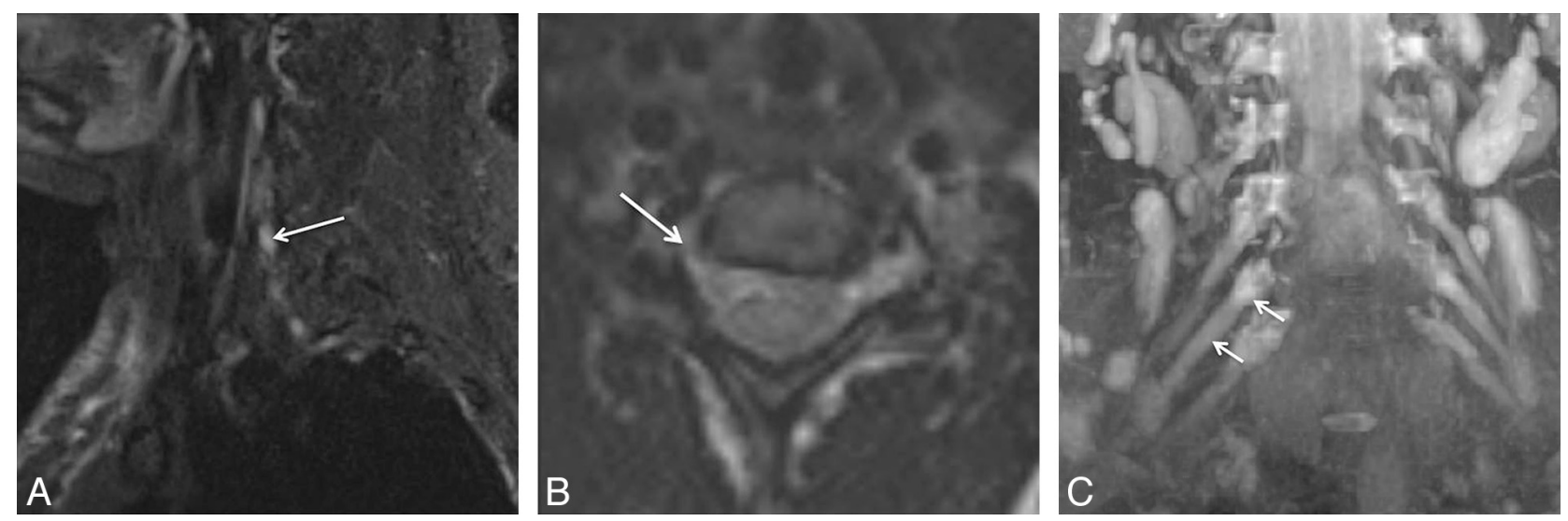

FIG 3. Isolated C6 radiculopathy. A 51-year-old woman with right arm pain and a tingling sensation, clinically suspected of having brachial plexitis versus radiculopathy. Sagittal STIR (A), axial T2 SPACE (B), and coronal 3D MIP STIR SPACE (C) images show an asymmetrically hyperintense and diffusely enlarged isolated C6 nerve root (arrows), corresponding to the markedly narrowed right C6 neural foramen. The findings are in keeping with cervical radiculopathy, in the setting of cervical spondylosis.
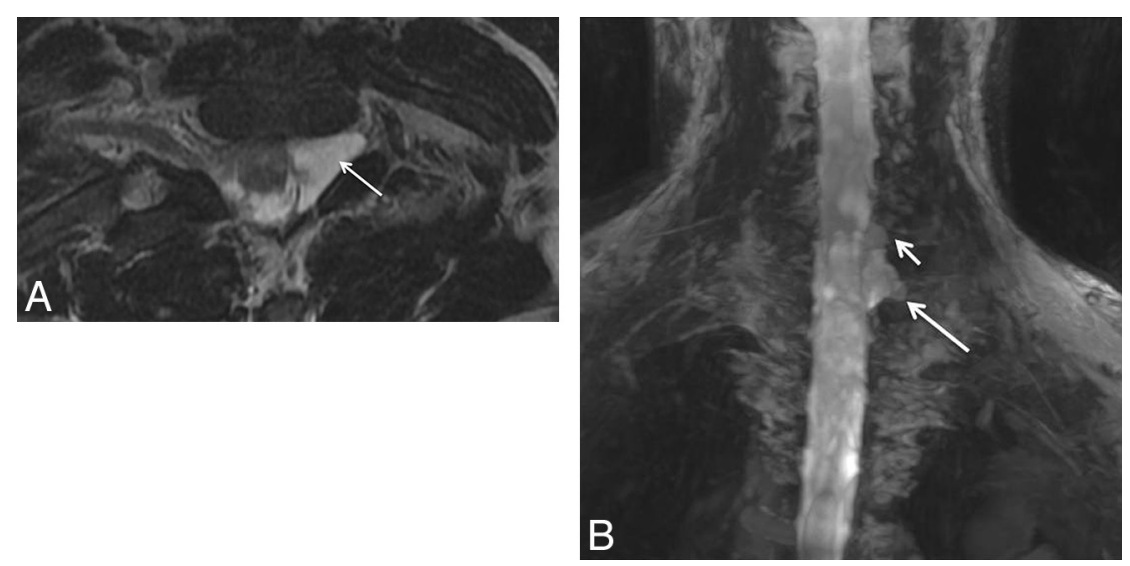

FIG 4. Nerve root avulsion. A 22-year-old man after a motor vehicle crash and ulnar-sided arm weakness. Axial T2-weighted $(A)$ and coronal MIP 3D STIR SPACE $(B)$ images show the avulsed left $\mathrm{Tl}$ nerve root (large arrows) and C8 nerve root (small arrow) with pseudomeningocele formation.

\section{ABNORMAL MRN FINDINGS IN THE BRACHIAL PLEXUS}

Abnormal brachial plexus findings may involve primary nerve findings with or without an associated anatomic variation, such as the accessory scalene muscle belly or intramuscular course; surrounding fat plane changes; perineural spaceoccupying lesions; cervical spondylosis or injury; regional denervation muscle changes; and, often, a combination of all these. ${ }^{16}$

Nerve findings include asymmetric T2 hyperintensity; enlargement; kinking of nerves by fibrosis/flattening by intramuscular course/entrapment by the mass lesions; and discontinuity/focal neuroma formation in injury. Sometimes, it may be difficult to differentiate stretch injury/brachial plexitis-related $\mathrm{T} 2$ signal and size abnormalities of the nerve roots from underlying long-standing cervical spondylotic changes in middle-aged and elderly subjects, especially if no prior comparison study is available. Generally, asymmetric T2 hyperintensity in the scalene triangle, sparing the most proximal segment of the nerve root and dorsal nerve root ganglion, usually distinguishes brachial plexopathy from cervical spondylosis-related nerve impingement/irritation, which involves the most proximal portion of the nerve within and imme- diately distal to the neural foramen; additionally, the abnormality in the latter case is usually restricted to the nerve corresponding to the most narrowed foramen (Fig 3).

3D images show the entire nerve extent and focal signal-intensity changes. Course deviations and size changes are easily appreciated by isotropic generation of the nerve image in its longitudinal plane; however, 3D images are less helpful in detecting fascicular abnormalities. While interpreting these nerve abnormalities, one should report isolated asymmetric mild T2 signal abnormalities as a nonspecific change which are particularly common in the authors' experience in C8 and $\mathrm{T} 1$ nerve roots. This $\mathrm{T} 2$ signal abnormality may be related to the magic angle effect or a manifestation of T2 anisotropy or may represent subclinical traction neuropathy. Loss of fat planes around all or part of a plexus reflecting fibrosis, tumor, or hemorrhage may be noted, depending on the clinical setting. Regional denervation muscle changes in the neck and shoulder are extremely helpful in indicating the nerve injury/ inflammation. These may range from edema-like T2 signal abnormalities in the initial stages of denervation to fatty replacement and atrophy in subacute and chronic stages. The degree of muscle fatty replacement and atrophy may also be helpful in guiding the surgical management in terms of nerve repair or tendontransfer procedures. ${ }^{1,2,4,5,7,8}$

\section{BRACHIAL PLEXUS INJURIES}

The most common cause of injury is motor vehicle crashes with or without fractures and dislocations of the cervical spine, or obstetric injuries. Other etiologies include sports injury, gunshot wound, rucksack injury, and iatrogenic traction injuries during anesthesia. From a surgical and prognostic point of view, the injuries are classified into 3 categories: preganglionic (Fig 4), postganglionic, and a combination of both. It is important to carefully 

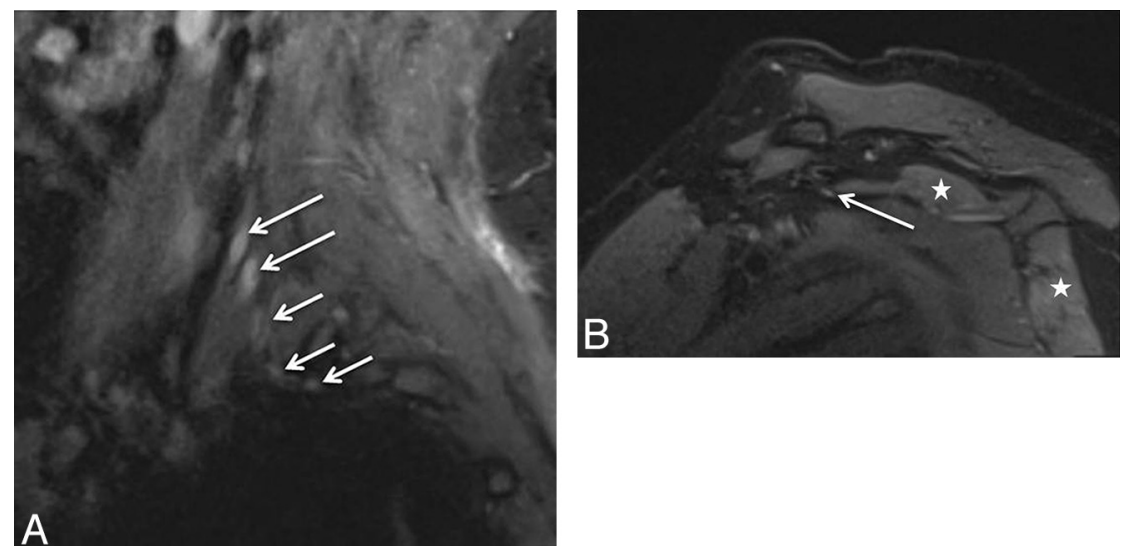

FIG 5. Stretch injury with suprascapular neuropathy. A 48-year-old man after a recent motor vehicle crash. Sequential sagittal STIR images $(A$ and $B)$ show asymmetrically enlarged and hyperintense $\mathrm{C} 5$ and $\mathrm{C} 6$ nerve roots (large arrows in $A$ ) relative to other normal nerve roots (small arrows). All the nerve segments are in continuity. Notice the hyperintense suprascapular nerve (large arrow in $B$ ) and mild denervation edema of the supraspinatus and infraspinatus muscles (stars).
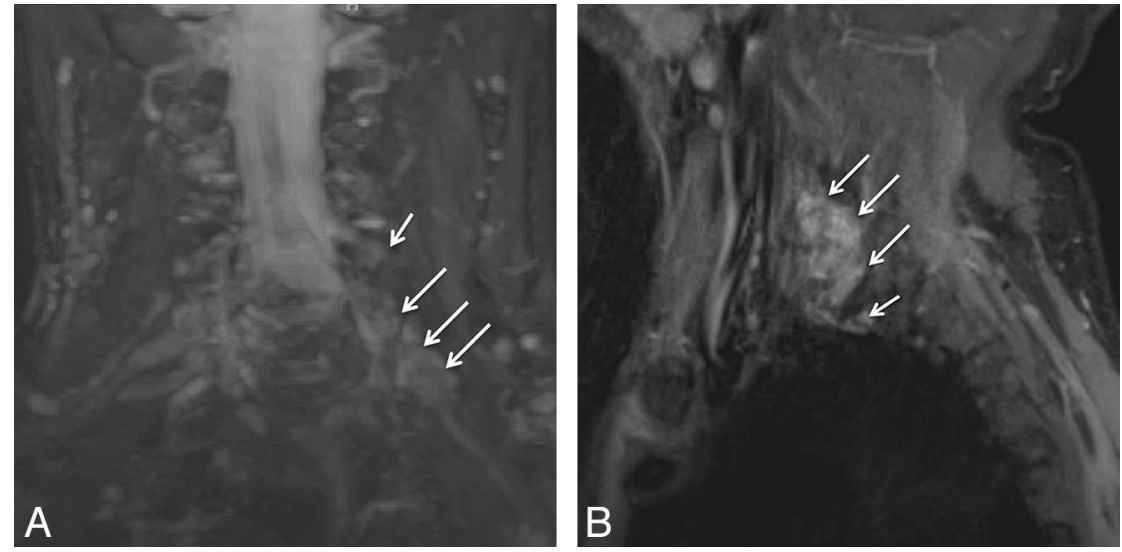

FIG 6. Multiple acute nerve root avulsions. A 51-year-old man with loss of function in the left upper extremity due to recent motor vehicle crash. Coronal MIP 3D STIR SPACE $(A)$ and sagittal STIR (B) images show avulsed C6, C7, and C8 nerve roots (arrows) with abnormal morphology due to hemorrhage and edema from a recent injury, obscuring clear details of the nerve roots. Notice the normal C5 nerve root (large arrows in $A$ ) and the normal 71 nerve root (small arrow in $B$ ).

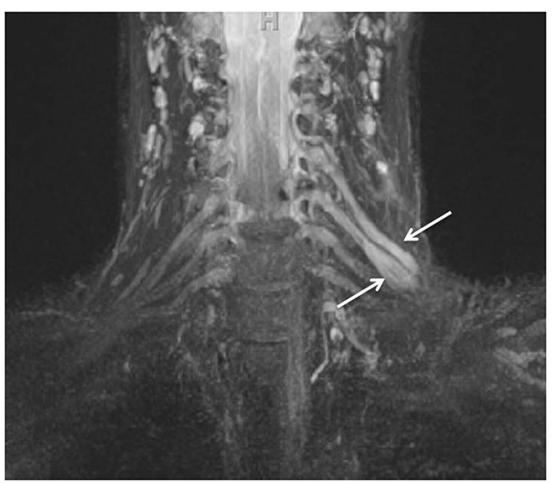

FIG 7. Neurotmesis. A 43-year-old woman with loss of function in the left upper extremity following recent neck surgery. Coronal MIP 3D STIR SPACE image shows severed, enlarged, and hyperintense $C 5$ and C6 nerve roots with distal end bulb neuromas (arrows), just proximal to the formation of the left upper trunk. The findings were confirmed on re-exploration, and the patient underwent immediate nerve transfer. look for the nerve roots from the spinal cord to the extraforaminal location because nerve root avulsion requires a major procedure, neurotization. Postganglionic lesions are further classified into lesions in continuity (requiring rehabilitation/neurolysis) and nerve discontinuity (requiring nerve repair/grafting). The overall spectrum of injuries ranges from neuropraxia/stretch injury (most common) to axonotmesis, partial neurotmesis with neuroma in continuity formation to complete nerve lacerations (neurotmesis)/ nerve root avulsions, the latter being the most severe injury. These injuries may concomitantly affect various upper limb nerves, such as the suprascapular, musculocutaneous, and axillary nerves.

MRN demonstrates the location of the nerve damage, depicts the nerve continuity with or without neuroma formation, or may show a completely disrupted/ avulsed nerve, thereby aiding in nerve-injury grading for preoperative planning and complementing the findings generated from electrodiagnostic studies (Figs 4-7). 3D images are useful to evaluate the entire extent of the injury and are great for demonstrating abnormalities to the referring physicians for optimal presurgical planning. Pre- and postganglionic injuries can also be differentiated by using EMG. Paraspinal muscle abnormality indicates that the injury is proximal to the brachial plexus trunks; however, an imaging study, such as a CT myelogram or $\mathrm{MRN}$, is required to differentiate incomplete from complete root avulsion or a spinal rootlet avulsion. A dural tear with formation of a pseudomeningocele may be seen on conventional MR imaging, but this finding is not pathognomonic for nerve root avulsion. MRN also shows another key finding - regional denervation muscle changes (Fig 5). The edema-like T2 signal intensity can appear within a few days, while contrast enhancement in the abnormal muscles has been shown to appear within 24 hours of the injury. Abnormal enhancement of paraspinal (especially multifidus) muscles has been shown to be an accurate indirect sign of root avulsion injury, which can also be evident on STIR images. As part of the protocol, T2 SPACE images should be used to assess spinal cord signal abnormality, which may be evident in approximately $20 \%$ of patients with preganglionic injuries as increased signal from cord edema or myelomalacia with or without decreased signal from blood product deposition. ${ }^{1,4,9,10,17}$

\section{INTERPRETATION PITFALLS}

Some interpretation pitfalls should be kept in mind. C5 nerves are the smallest of all nerves but are generally symmetrical on both 

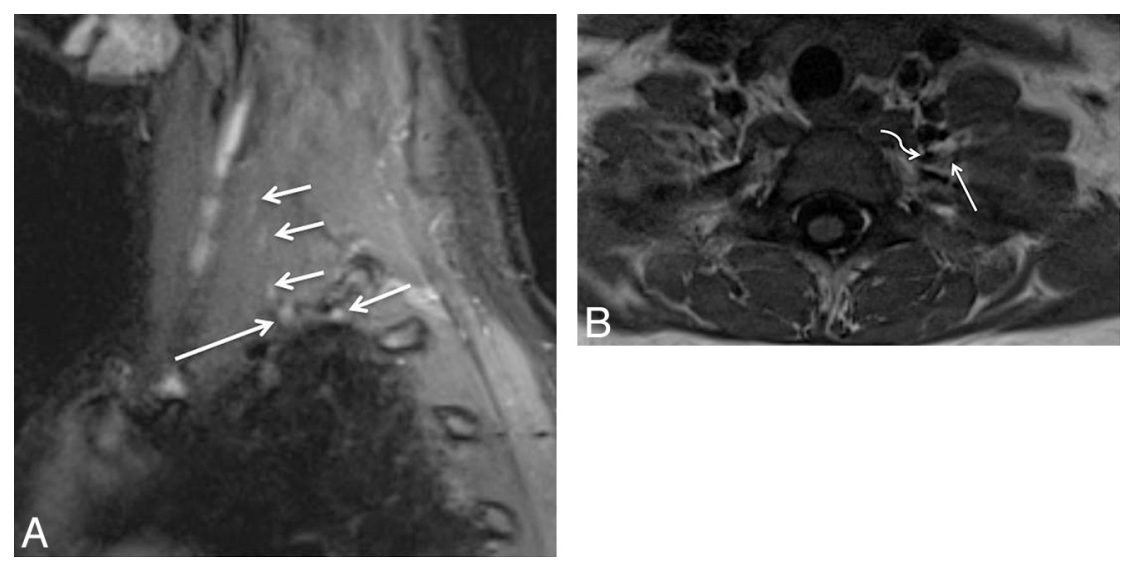

FIG 8. TOS with C8 neuropathy. A 17-year-old girl (trumpet player) with left arm and hand weakness in an ulnar distribution, exacerbated by shoulder abduction. Sagittal STIR $(A)$ image shows an asymmetrically enlarged and hyperintense C8 nerve root (large arrow) compared with the other normal nerve roots (small arrows). Notice minimal asymmetric hyperintensity of the T1 nerve root, a common nonspecific finding. Axial T1-weighted $(B)$ MR image through the lower neck shows a prominent flow void (curved arrow) indenting the left C 8 nerve root (large arrow). The EMG findings were normal in this case. On surgery, the flow void turned out to be a prominent branch of the left thyrocervical trunk, which was ligated. C8 neurolysis was also performed. The patient's symptoms completely resolved following surgery. types of TOSs: disputed (most common), vasculogenic (arterial, venous, or both), true neurogenic (nerve), combined (neurovascular), and droopy shoulder syndrome. ${ }^{18,19}$ Disputed form presents with only sensory symptoms and is considered a controversial entity. Vasculogenic TOS occurs most commonly in young athletes with frequent hyperabduction or overhead activities, due to compression of axillary or subclavian vessels. The compression may be static or dynamic (positional) and may result in stenosis, thrombosis, and aneurysm formation (also referred to as the Paget-von Schrotter syndrome). Shoulder and arm pain with swelling and discoloration/temperature changes of the affected hand are possible symptoms. True neurogenic TOS (Gilliatt-Sumner hand; minority of TOS cases) usually results from compression of the lower trunk of the plexus by anatomic variations as

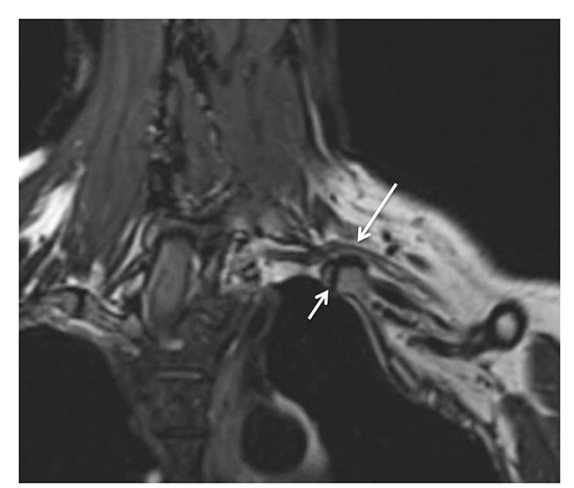

FIG 9. Thoracic outlet syndrome. A 55-year-old woman with weakness of the left upper extremity with tingling in the hand. Coronal T2 SPACE image shows the deviated path of the C8 nerve root with abnormal flattening (arrow) due to pseudoarthrosis between the left cervical rib and first rib, which was subsequently proved on the surgery. The patient improved after surgery.

sides. There may be decreased diagnostic accuracy in acute injuries due to partial obscuration of the nerve detail by hemorrhage and edema and in subacute injuries from an otherwise avulsed nerve root with perineural scarring, mimicking the appearance of a partially intact nerve root. Abnormal T2 signal intensity within the nerves may persist for prolonged periods from months to years. Therefore, one should look for regional muscle status and the clinical picture to determine the acuity of the disease process. Abnormal T2 signal intensity similar to a stretch injury or brachial neuritis may also be caused by underlying cervical spondylosis and neural foraminal stenosis, with potential imaging differences as discussed above. ${ }^{4}$

\section{THORACIC OUTLET SYNDROME}

Kinnier Wilson first described TOS in 1900 as a syndrome involving compression of a neurovascular bundle passing between the anterior scalene and middle scalene muscles. There are various described above. It is more common in women and presents with medial arm pain and ulnar-sided hand and forearm sensory dysfunction (tingling) and weakness (Fig 8). Droopy shoulder syndrome is also common in young women presenting with shoulder pain and paresthesias. On radiographs, the second thoracic vertebra may be seen on the lateral view. Drooped shoulders are usually bilateral and may cause stretching of the brachial plexus segments. Other subjects likely to develop TOS are those who have neck nonergonomic postures for extended periods. The diagnosis of TOS is traditionally based on results of clinical evaluation, including various dynamic maneuvers such as elevation of the arm. However, diagnosis based on clinical examination alone is often difficult. Additionally, the proximal location of the nerve roots and subtle demyelinating changes developing in the initial stages of the disease limit the ability of an electromyographer to detect mild or early TOS.

As mentioned above, minimal T2 hyperintensity of the C8 and $\mathrm{T} 1$ nerve roots is commonly seen as a nonspecific finding; altered T2 signal intensity extending into the lower trunk or associated nerve enlargement or both generally correlate with clinical findings of TOS. Although uncommon, involvement of the middle trunk or kinking of the nerve roots by a fibrous band or flattening due to an intramuscular course of the nerve roots, impingement by anomalous vessels, narrowing of the interscalene space by a hypertrophied muscle, or cervical ribs or costocostal pseudoarthrosis impinging the nerves may be seen as abnormal findings (Fig 9). ${ }^{4,18,20-23}$ Simultaneous depiction of the causative lesion, such as a cervical rib and associated displacement of the nerve with signal abnormality, is possible on curved reconstructions generated from isotropic 3D imaging (Fig 9). The treatment of TOS may be rehabilitation or surgery by anterior scalenectomy and anomalous rib resection. Although MRN is frequently used for presurgical planning, it may also preclude the suspected diagnosis of TOS, when it demonstrates a completely normal course injuries from traffic collisions and those who use computers in 

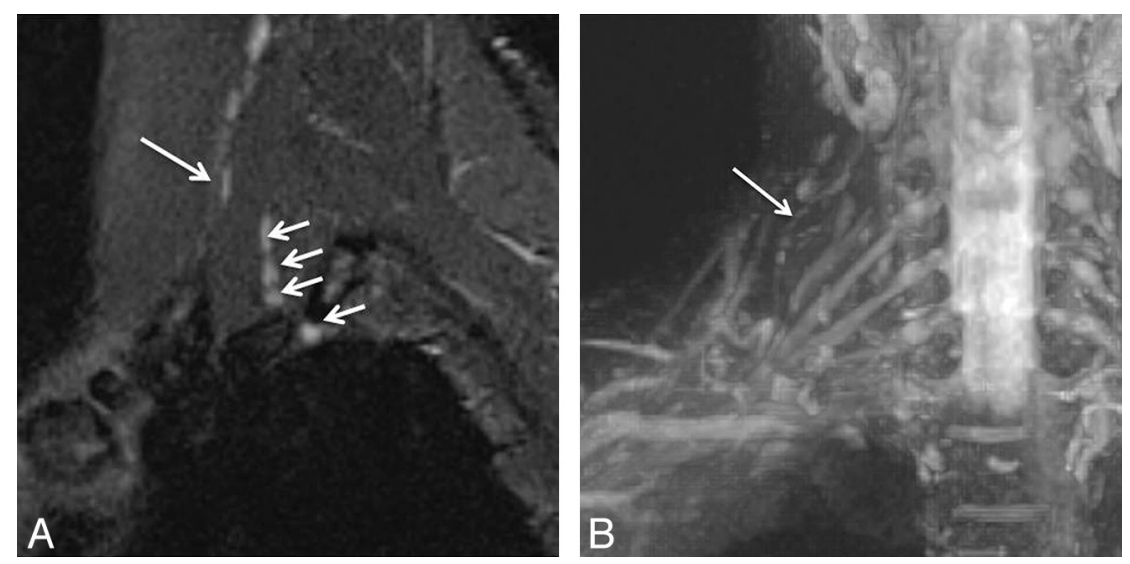

FIG 10. Intramuscular course and entrapment of the $C 5$ nerve root. A 48-year-old man after a recent motor vehicle crash. Sagittal STIR $(A)$ and coronal MIP 3D STIR SPACE $(B)$ images show the intramuscular course of the $C 5$ nerve root with relatively anterior and lateral positioning and mild flattening (large arrow) compared with the other brachial plexus nerve roots (small arrows). The patient had no symptoms referable to TOS pathology.

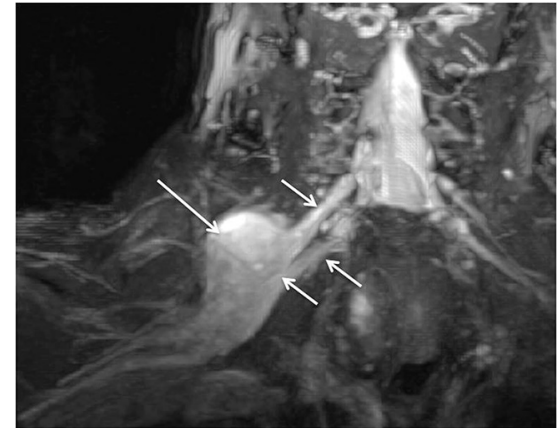

FIG 11. Malignant peripheral nerve sheath tumor. A 56-year-old man with right shoulder and arm pain. Coronal MIP 3D STIR SPACE image shows enlarged right C5 and C6 nerve roots and upper trunk (small arrows) with a surgically proved malignant nerve sheath tumor (large arrow).

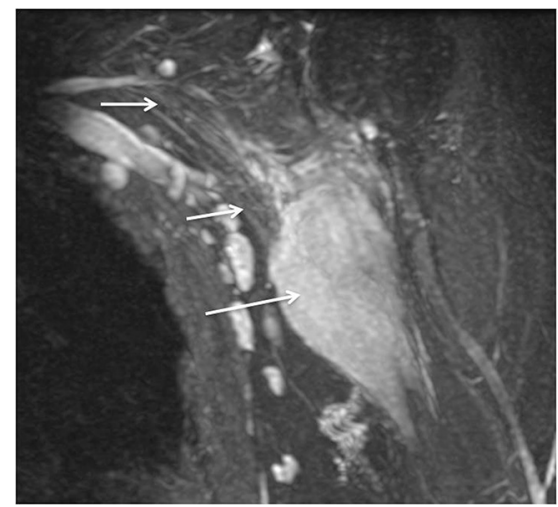

FIG 12. Lymphoma involving the terminal branches of the left brachial plexus. A 59-year-old man with left arm pain and weakness. Coronal MIP 3D STIR SPACE image shows mildly hyperintense peripheral nerve branches of the left brachial plexus (small arrows); distally encased by the B-cell lymphoma mass lesion (large arrow).

and caliber for all nerve segments that are under suspicion, thereby presenting evidence against unnecessary surgery. A variant intermuscular course might not always be symptomatic (Fig 10). MRN is also useful for detection of focal fibrosis causing

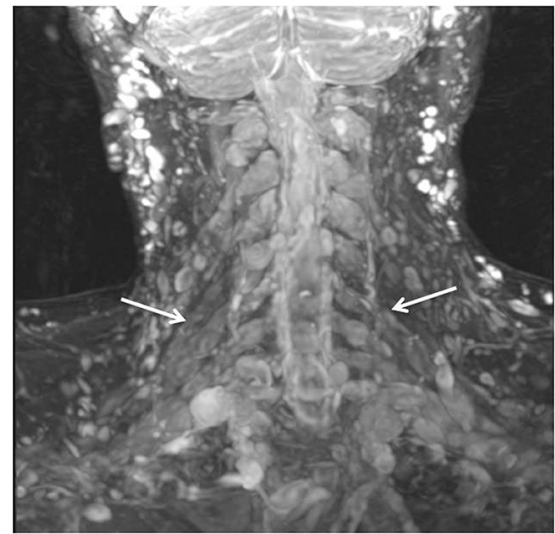

FIG 13. Neurofibromatosis type 1. Coronal MIP 3D STIR SPACE image in a 24-year-old woman shows numerous cutaneous and subcutaneous hyperintense peripheral nerve sheath tumors along with diffuse nodular thickening of bilateral brachial plexus segments (arrows).

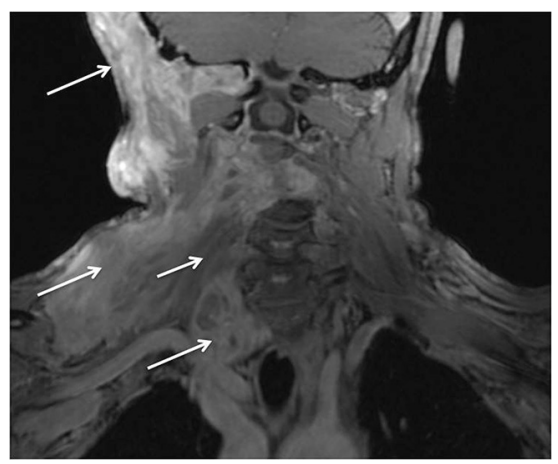

FIG 14. Plexiform neurofibroma in neurofibromatosis type-1 (NF-1). A 36-year-old man with known NF-1 presenting with right shoulder and neck pain with weakness of the arm. Coronal postcontrast fat-suppressed Tl-weighted image shows a large infiltrative mass showing a "bag of worms" appearance (large arrows), encasing the brachial plexus (small arrow), in keeping with a plexiform neurofibroma.

re-entrapment of the nerves, with persistent enlargement and T2 signal abnormality of the nerves in failed cases of thoracic outlet surgery. 

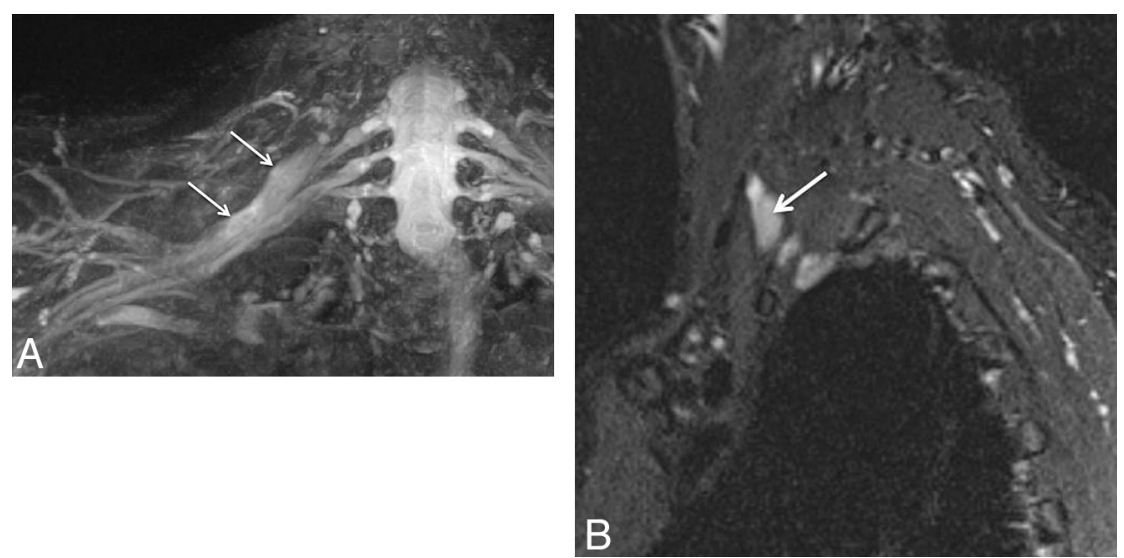

FIG 15. Lymphoma. A 65-year-old man with a history of lymphoma who presented with newonset weakness of the right upper extremity. Coronal MIP 3D STIR SPACE $(A)$ and sagittal STIR $(B)$ images show fusiform enlargement of the right $C 7$ nerve root (arrows) in keeping with lymphomatous involvement.

\section{TUMOR AND TUMOR VARIANTS}

Various space-occupying lesions, such as hematoma, nerve sheath tumors, benign tumors, primary or secondary tumors of perineural spaces, and various tumor variants (diffuse polyneuropathies) may involve the different elements of the brachial plexus. Benign peripheral nerve sheath tumors include neurofibroma (unencapsulated, infiltrates the fascicles), schwannoma (encapsulated, displaces the nerve fascicles), and ganglioneuroma (an elongated lobulated lesion along the ganglion chain). The peripheral nerve sheath tumors show classic MR imaging signs of the split fat sign, target sign, fascicular sign, and tail sign. Malignant peripheral nerve sheath tumors occur less commonly, and these lesions are frequently large $(>5$ $\mathrm{cm}$ ), present with worsening pain or new neurologic deficits (Fig 11), and demonstrate a heterogeneous appearance and enhancement. Current 3D MR imaging techniques, including contrast enhancement features, beautifully depict the lesion and its topographic characteristics; however, the imaging findings may not accurately indicate the benignity of the lesion. In the future, it is expected that diffusion tensor imaging will likely play a useful role in differentiation of these lesions by providing a functional insight into the lesions.

Nevertheless, local tissue invasion and metastasis are helpful signs to suggest malignancy. High $\left[{ }^{18} \mathrm{~F}\right]$ FDG PET uptake (standard uptake value $>3-4$ ), especially with delayed and persistent uptake of glucose, may be useful in indicating the aggressive/malignant behavior of the lesion; however, low uptake does not exclude malignancy. Benign mass lesions causing brachial plexopathy may include lipoma, fibromatosis, perineural cyst, hemangioma, lymphangioma, and so forth. Common malignancies affecting the brachial plexus include pancoast tumor, breast metastasis, lymphoma (neurolymphomatosis), and metastatic lymphadenopathy (Fig 12). ${ }^{1,4,7,8,12,24}$ These lesions may be focal or infiltrating and usually present with supraclavicular lower plexus (C7-T1) involvement. Diffuse involvement may be seen with lesions in neurocutaneous syndromes, such as plexiform neurofibroma, plexiform schwannoma, or neurolymphomatosis (Figs 13-15), as well as with tumorlike conditions such as multifocal motor and sensory neuropathy; hereditary neuropathy, such as Charcot-MarieTooth disease and chronic inflammatory demyelinating neuropathy; and so forth. ${ }^{12}$ Charcot-Marie-Tooth disease, especially type 1A (demyelinating type), and neurocutaneous syndromes may lead to marked diffuse thickening of nerves. MR imaging, especially whole-body imaging, is being used to assess and follow-up the disease burden in cases of neurocutaneous syndromes, such as schwannomatosis, or to survey the regional area for any growing lesion if the patient presents with new or recalcitrant symptoms. ${ }^{25,26}$

\section{RADIATION NEUROPATHY}

Patients presenting with recurrent symptoms following radiation treatment for malignancy may have tumor recurrence or radiation plexopathy. Tumor recurrences usually occur within 1 year following the treatment and lead to lower supraclavicular plexus involvement (predominately C8, T1, and the lower trunk). Radiation plexopathy may occur from a few months to 20 years from the date of treatment (5\%-10\% incidence) and involves the infraclavicular plexus (Fig 16). Although symptoms of pain, paresthesias, and motor weakness can be seen in both scenarios, EMG shows characteristic myokymic discharges in approximately $30 \%$
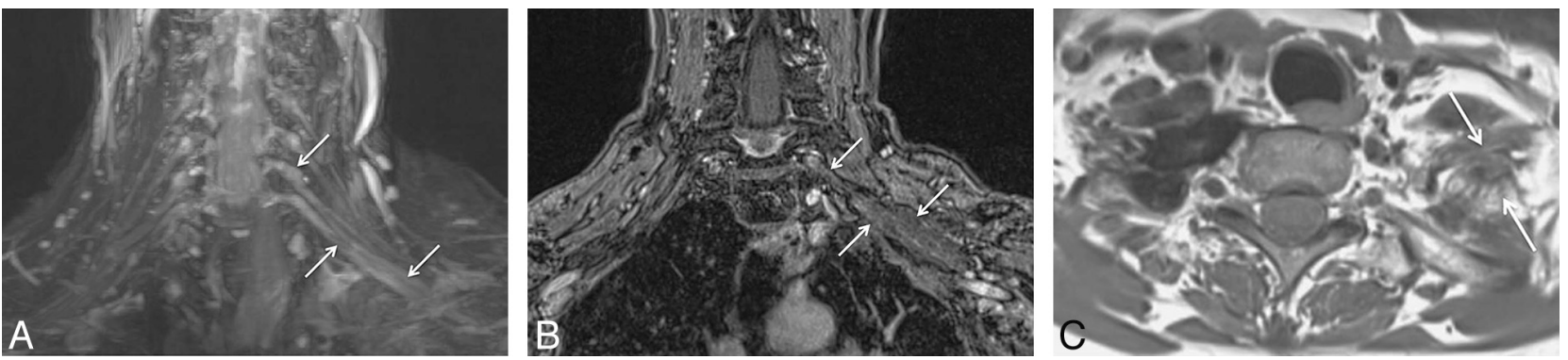

FIG 16. Radiation plexopathy. A 67-year-old woman with a history of left breast carcinoma and radiation treatment 20 years ago who presented with weakness and pain in the left upper extremity. Coronal MIP 3D STIR SPACE (A) image shows diffusely enlarged and abnormally hyperintense left C 8 and T1 nerve roots and lower trunk (arrows). Corresponding coronal fat-suppressed postcontrast T1-weighted (B) image shows no focal enhancement (arrows) to suggest recurrent disease. Axial non-fat-suppressed T1-weighted $(C)$ image shows perineural radiation fibrosis encasing the left C8/TI nerve roots and the lower trunk of the brachial plexus (arrows). 


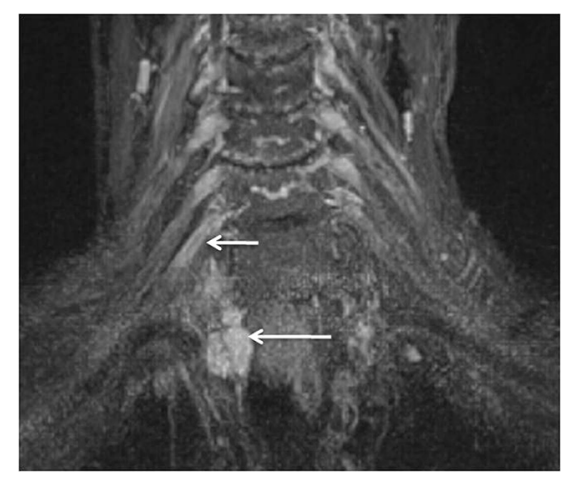

FIG 17. Radiation neuropathy. An 81-year-old woman, previously treated for right breast carcinoma, who presented with right arm and neck pain. Coronal 3D STIR SPACE image shows a focal mass (large arrow) encasing the right $\mathrm{Tl}$ nerve root. Additionally, there are mild enlargement and hyperintensity of the right C8 nerve root (small arrow).

of cases, usually not seen with tumor infiltration. MRN shows a focal or diffuse enhancing mass lesion in case of tumor recurrence, with asymmetric enlargement of the plexus (Fig 17). ${ }^{12,27}$ In postradiation patients, the abnormality is generally geographic in the radiation field and diffuse and symmetric without focal masslike enhancement on contrast examinations. Postradiation fibrosis may also be seen as T1 and T2 hypointense fat stranding with distortion and kinking of the nerve segments (Fig 16).

\section{BRACHIAL PLEXITIS (NEURALGIC AMYOTROPHY OR PARSONAGE TURNER SYNDROME)}

Idiopathic brachial plexitis is seen with an incidence of 1.6/ 100,000 . Proposed etiologies include immune versus inflammatory. It affects young and middle-aged patients, males more than females, and may be bilateral in up to $30 \%$ of patients. Patients present with sudden onset of severe constant pain in the neck, shoulder, or upper arm, which within a few weeks, is followed by profound weakness and atrophy of the regional muscles. The most frequently affected muscles are the serratus anterior, supraspinatus, and infraspinatus muscles. While multiple nerves may be affected, for unknown reasons, the ulnar nerve is rarely

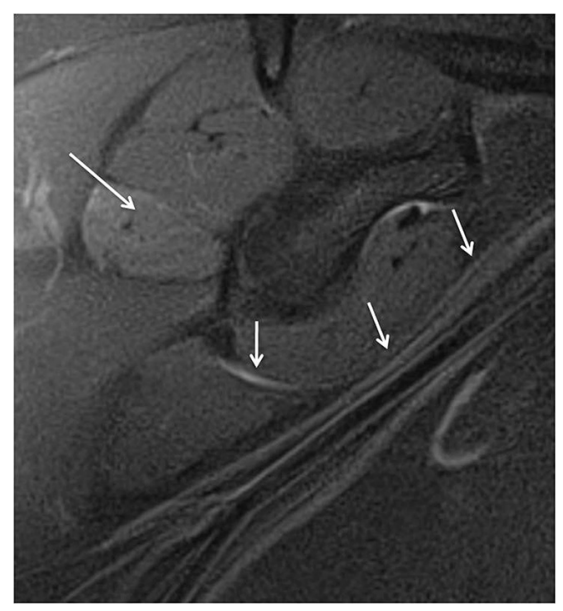

FIG 19. Brachial and axillary neuritis. A 43-year-old woman with right shoulder pain. Sagittal fat-suppressed T2-weighted image shows an abnormally hyperintense axillary nerve (small arrows) and denervation edema in the teres minor muscle (large arrow).

affected. MRN may show diffusely enlarged and hyperintense nerves and scattered muscle denervation changes around the shoulder joint (Figs 18 and 19). ${ }^{1,28,29}$ Treatment is pain control and rehabilitation. The therapeutic benefit of steroids is controversial.

\section{PERIPHERAL BRANCH NERVES OF BRACHIAL PLEXUS Suprascapular Nerve (C5, C6)}

The suprascapular nerve is a mixed motor-sensory nerve supplying the shoulder joints and the supraspinatus and infraspinatus muscles. It arises from the upper trunk of the brachial plexus and travels laterally in the posterior triangle of the neck to enter the supraspinatus fossa through the suprascapular notch of the scapula, which is covered by the transverse scapular ligament. This fibro-osseous tunnel makes it vulnerable to entrapment due to mass lesions such as paralabral cysts, varicosities, variant ligaments or muscle insertions, rotator cuff tears, and tumors. It courses posteroinferiorly, passing through the spinoglenoid notch to reach the infraspinatus fossa. It supplies the supraspina-
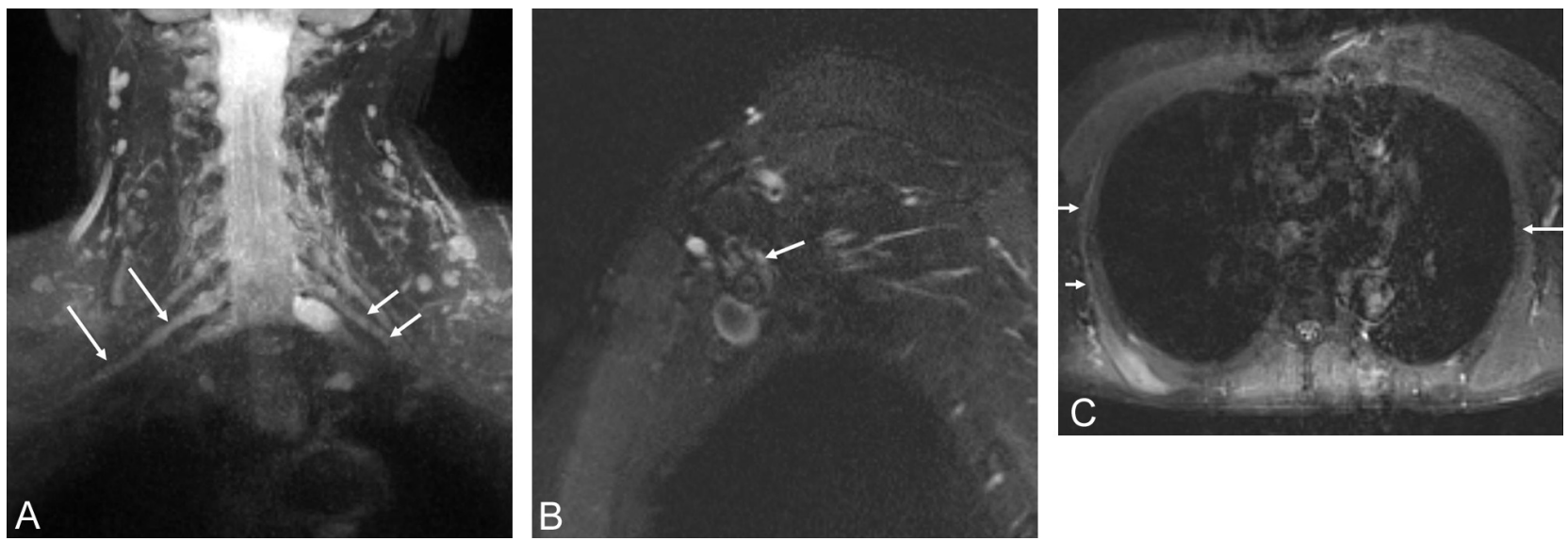

FIG 18. A, Brachial neuritis with long thoracic nerve neuritis. A 54-year-old man with right shoulder pain and right scapular winging for a few months. Coronal 3D STIR SPACE image shows the asymmetrically hyperintense and enlarged C7 nerve (large arrows). The abnormality extended into the middle trunk and its divisions (not shown). Notice normal left C7 nerve root (small arrows). Sagittal STIR (B) image shows the abnormally enlarged and hyperintense long thoracic nerve along its course (arrow). Axial STIR (C) image through the chest shows atrophy and edema like signal of the right serratus anterior muscle (small arrows). Notice the normal left serratus anterior muscle (large arrow). 

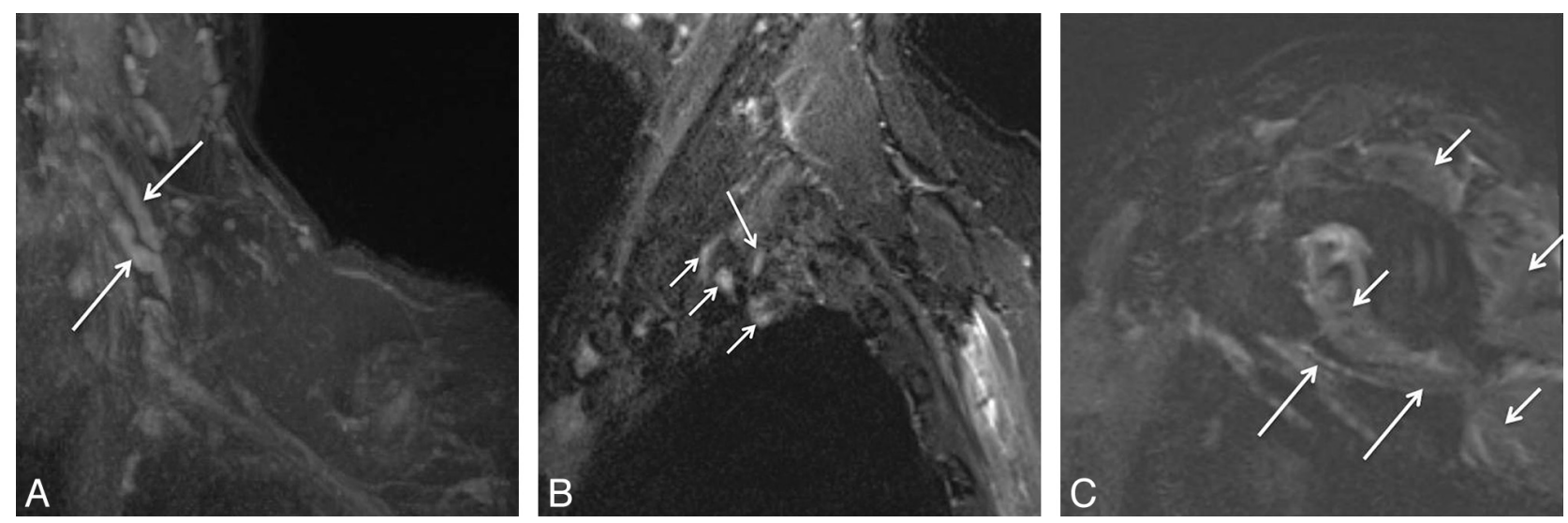

FIG 20. Neurotmesis with suprascapular nerve severance and axillary neuropathy. A 46-year-old man with a dirt bike injury. Coronal MIP 3D STIR SPACE (A) image shows complete disruption of the left C5 and C6 nerve roots (arrows), proximal to the formation of the upper trunk. Sagittal STIR (B) image shows an enlarged and hyperintense suprascapular nerve proximal to its disruption (large arrow). Also note hyperintense trunks of the brachial plexus (small arrows). Further lateral sagittal STIR (C) image shows denervation changes in the rotator cuff (small arrows) muscles ( $\mathrm{C} 5$ and $\mathrm{C} 6$ distribution) and an abnormally hyperintense but continuous axillary nerve (large arrows).
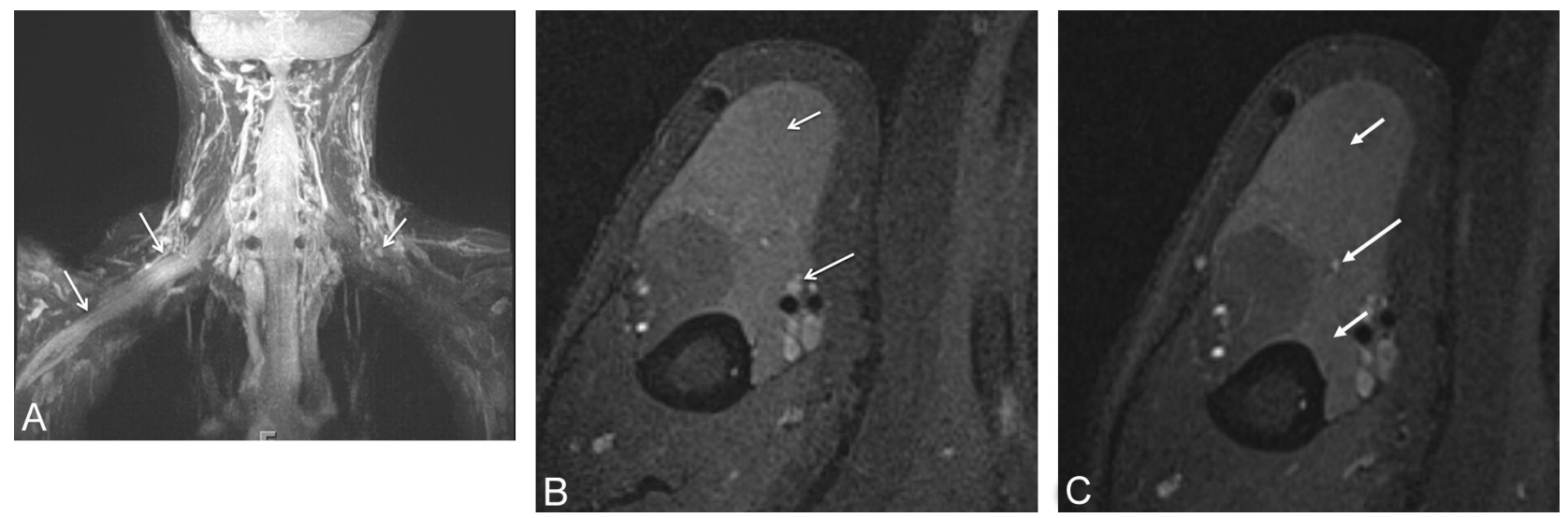

FIG 21. $A$, Stretch Injury to right sided brachial plexus. Coronal 3D STIR SPACE (A) image shows diffusely enlarged right sided brachial plexus (large arrows) following a recent clavicular fixation for fracture. Notice normal left brachial plexus (small arrows). The patient improved over a few months of expectant treatment. $B$ and $C$, Stretch injury of the C6 nerve root and musculocutaneous nerve. A 60-year-old man with weakness of the right biceps and brachialis muscles following a recent injury. Axial T2 SPAIR (B) image through the proximal right arm shows an abnormally hyperintense and enlarged musculocutaneous nerve (large arrow) with denervation changes in the biceps and brachialis muscles (small arrows).

tus muscle at the suprascapular notch and the infraspinatus muscle distally. Therefore, if a compressing lesion is located around the suprascapular notch, both the supraspinatus and the infraspinatus muscles are denervated. When a compressing lesion is located around the spinoglenoid notch, only the infraspinatus is denervated. Patients may present with shoulder pain and weakness in abduction (supraspinatus) and external rotation (infraspinatus). EMG may confirm the nerve abnormality. On MRN, the normal suprascapular nerve is generally not seen, while in abnormal cases, the nerve can be seen throughout its course and its continuity; entrapment by a mass lesion and regional denervation muscle change are evident. In brachial plexitis cases, MRN shows the abnormal nerve and coexistent brachial plexus abnormalities involving C5, C6, and the upper trunk in variable combinations (Figs 5 and 20). ${ }^{11}$ Treatment is rehabilitation versus surgical neurolysis and release of the transverse scapular ligament.

\section{Axillary Nerve (C5, C6)}

The axillary nerve is a mixed motor-sensory nerve, which arises in the axilla from the posterior cord of the brachial plexus. The nerve fibers originate from the C5 and C6 roots, middle trunk, and its posterior division. The fibers continue to contribute to the upper trunk and posterior cord to finally form the axillary nerve. The axillary nerve courses through the axilla anterior to the subscapularis muscle and behind the axillary artery to the inferior aspect of the shoulder joint along with circumflex humeral vessels to terminate in the quadrilateral space. After exiting the quadrilateral space, the nerve finally terminates in anterior, posterior, and collateral branches to the long head of the triceps muscle. The anterior or upper branch winds around the surgical neck of the humerus and terminates after supplying the deltoid muscle and adjacent skin. The posterior or inferior branch supplies the teres minor, posterior belly of deltoid muscle, and adjacent skin.

The nerve can be involved with brachial neuritis or brachial plexus injuries or it may be injured in isolation during shoulder dislocation, humeral fracture, penetrating injury, rotator cuff surgery, or improper crutch use. The patient may have weakness of shoulder flexion, abduction, and external rotation as well as lat- 
eral shoulder sensory abnormalities. EMG may indicate the nerve abnormality. Treatment includes rehabilitation or surgery. On MRN examinations, the axillary nerve is often seen surrounded by normal fat, without focal enlargement or a mass. Abnormal nerve signal, size, and/or associated brachial plexopathy may be evident on high-resolution images (Figs 19 and 20). Muscle denervation changes in the teres minor and/or deltoid muscles confirm the nerve pathology. ${ }^{2}$ Isolated denervation change of the teres minor muscle is commonly seen as an isolated finding in patients with frozen shoulder, probably related to periligamentous edema causing inflammation of the supplying branch of the axillary nerve. ${ }^{30}$

\section{Musculocutaneous Nerve (C5-C7)}

The musculocutaneous nerve is a mixed motor-sensory nerve, which arises from the lateral cord of the brachial plexus at the lower border of the pectoralis major muscle. Its fibers originate from the $\mathrm{C} 5, \mathrm{C} 6$, and $\mathrm{C} 7$ nerve roots. It travels through the axilla and penetrates the coracobrachialis muscle and then passes between the biceps and brachialis muscles. Just above the elbow, it pierces the deep fascia lateral to the tendon of the biceps and continues as the lateral cutaneous nerve of the forearm. It innervates the coracobrachialis, biceps, and brachialis muscles.

The nerve is injured less frequently; however, it may be compressed between the biceps aponeurosis and brachialis fascia or have traction injuries due to shoulder dislocation, proximal humerus fracture, weight-lifting injuries, and sometimes during anesthesia. Neuropathy as part of brachial plexopathy may also result. EMG/nerve conduction study findings are usually positive. Although the normal nerve is isointense and is barely visible, MRN shows the abnormal nerve as T2 hyperintensity or enlargement, with or without associated brachial plexopathy involving the lateral cord and C5-C7 nerve roots (Fig 21). The denervation muscle changes may be observed in the coracobrachialis, biceps, and brachialis muscles. In traction injuries, MRN can confirm the nerve continuity, guiding the treatment. ${ }^{2,31,32}$

\section{Long Thoracic Nerve (C5-C7)}

The long thoracic nerve is a motor nerve with a single muscle supply and is a proximal branch of the brachial plexus that is formed by C5, C6, and C7 nerve roots. It originates from the posterior aspect of the plexus and passes through the middle scalene muscle. It then courses along the chest wall on the outer surface of the serratus anterior muscle, innervating the muscle along its path.

The nerve can be injured by traction from a fall, a motor vehicle crash, axillary lymph node dissection, mastectomy, or sports injury, or it may be entrapped within the middle scalene muscle or by a chest wall mass. It is also the most common nerve involved with brachial plexitis (Fig 18). Patients present with dull shoulder pain and a laterally winged scapula with weakness in overhead arm activity (ie, raising the arm in front of the body). The diagnosis can be confirmed by an EMG of the serratus anterior muscle. Because the nerve is very small and difficult to see on MRN, the role of imaging is to confirm the respective muscle denervation changes in a clinically confusing case. ${ }^{2}$ An abnormal nerve may be occasionally seen on MRN along with denervation changes in the serratus anterior muscle. Another important role of imaging is to exclude a neoplasm in a patient without a history of trauma or brachial plexitis. Treatment is rehabilitation or surgery.

\section{CONCLUSIONS}

The brachial plexus is anatomically complex and may be involved in a variety of pathologies, leading to significant morbidity. MRN plays a significant role in the diagnostic algorithm of plexus pathologies, aiding in early diagnosis and patient management.

Disclosures: Avneesh Chhabra—RELATED: research grants from Siemens AG and Integra Life Sciences and the support of a GE-AUR Fellowship, UNRELATED: Consultancy: Siemens, Comments: MSK CAD system development, Travel/Accommodations/Meeting Expenses Unrelated to Activities Listed: Siemens, Integra, GE-AUR. John A. Carrino- grant from Siemens Medical Systems to study MR neurography, UNRELATED: Consultancy: Quality Medical Metrics, Grants/Grants Pending: Toshiba Medical. Filippo Del Grande-institutional grant from Siemens, UNRELATED: Stock/Stock Options: GE Healthcare, Comments: shareholder of approximately $\$ 6100$.

\section{REFERENCES}

1. Du R, Auguste KI, Chin CT, et al. Magnetic resonance neurography for the evaluation of peripheral nerve, brachial plexus, and nerve root disorders. J Neurosurg 2010;112:362-71

2. Martinoli C, Gandolfo N, Perez MM, et al. Brachial plexus and nerves about the shoulder. Semin Musculoskelet Radiol 2010;14:523-46

3. Tagliafico A, Succio G, Emanuele Neumaier C, et al. MR imaging of the brachial plexus: comparison between 1.5-T and 3-T MR imaging: preliminary experience. Skeletal Radiol 2011;40:717-24

4. Chhabra A, Lee PP, Bizzell C, et al. 3 Tesla MR neurography: technique, interpretation, and pitfalls. Skeletal Radiol 2011;40:1249-60

5. van Es HW, Bollen TL, van Heesewijk HP. MRI of the brachial plexus: a pictorial review. Eur J Radiol 2010;74:391-402

6. Sureka J, Cherian RA, Alexander M, et al. MRI of brachial plexopathies. Clin Radiol 2009;64:208-18

7. Perić S, Lavrnić S, Basta I, et al. Significance of magnetic resonance imaging in differential diagnosis of nontraumatic brachial plexopathy. Vojnosanit Pregl 2011;68:327-31

8. Iyer VR, Sanghvi DA, Merchant N. Malignant brachial plexopathy: a pictorial essay of MRI findings. Indian $J$ Radiol Imaging 2010;20:274-78

9. Smith AB, Gupta N, Strober J, et al. Magnetic resonance neurography in children with birth-related brachial plexus injury. Pediatr Radiol 2008;38:159-63

10. Medina LS, Yaylali I, Zurakowski D, et al. Diagnostic performance of MRI and MR myelography in infants with a brachial plexus birth injury. Pediatr Radiol 2006;36:1295-99

11. Chalian M, Faridian-Aragh N, Soldatos T, et al. High-resolution 3T MRN of suprascapular neuropathy. Acad Radiol 2011;18:1049-59

12. Thawait SK, Chaudhry V, Thawait GK, et al. High-resolution MRN of diffuse peripheral nerve lesions. AJNR Am J Neuroradiol 2011;32:1365-72

13. Vargas MI, Viallon M, Nguyen D, et al. New approaches in imaging of the brachial plexus. Eur J Radiol 2010;74:403-10

14. Tagliafico A, Succio G, Neumaier CE, et al. Brachial plexus assessment with three-dimensional isotropic resolution fast spin echo MRI: comparison with conventional MRI at 3.0 T. $\mathrm{Br}$ J Radiol 2011;85:e110-16

15. Tagliafico A, Calabrese M, Puntoni M, et al. Brachial plexus MR imaging: accuracy and reproducibility of DTI-derived measurements and fibre tractography at 3.0-T. Eur Radiol 2011;21: $1764-71$

16. Viallon M, Vargas MI, Jlassi $\mathrm{H}$, et al. High-resolution and functional magnetic resonance imaging of the brachial plexus using an isotro- 
pic 3D T2 STIR (short term inversion recovery) SPACE sequence and diffusion tensor imaging. Eur Radiol 2008;18:1018-23

17. Yoshikawa T, Hayashi N, Yamamoto S, et al. Brachial plexus injury: clinical manifestations, conventional imaging findings, and the latest imaging techniques. Radiographics 2006;26(suppl 1):S133-43

18. Demondion $X$, Herbinet $P$, Van Sint Jan S, et al. Imaging assessment of thoracic outlet syndrome. Radiographics 2006;26:1735-50

19. Al-Shekhlee A, Ktirji B. Spinal accessory neuropathy, droopy shoulder, and thoracic outlet syndrome. Muscle Nerve 2003;28:383-85

20. Sabapathy SR, Venkatramani H, Bhardwaj P. Pseudarthrosis of cervical rib: an unusual cause of thoracic outlet syndrome. J Hand Surg Am 2010;35:2018-21

21. Aralasmak A, Karaali K, Cevikol C, et al. MR imaging findings in brachial plexopathy with thoracic outlet syndrome. AJNR Am J Neuroradiol 2010;31:410-17

22. Parker EE, Glastonbury CM. MR imaging of the thoracic inlet. Magn Reson Imaging Clin N Am 2008;16:341-53, x

23. Desurkar A, Mills K, Pitt M, et al. Congenital lower brachial plexus palsy due to cervical ribs. Dev Med Child Neurol 2011;53:188-90

24. Chaturvedi A, Singh JP, Rastogi V. MRI diagnosis of neurolymphomatosis of the brachial plexus. Neurol India 2008;56:485-86
25. Chhabra A, Blakely J. Whole-body imaging in schwannomatosis. Neurology 2011;76:2035

26. Cai W, Kassarjian A, Bredella MA, et al. Tumor burden in patients with neurofibromatosis types 1 and 2 and schwannomatosis: determination on whole-body MR images. Radiology 2009;250: 665-73

27. Harper CM Jr, Thomas JE, Cascino TL, et al. Distinction between neoplastic and radiation-induced brachial plexopathy, with emphasis on the role of EMG. Neurology 1989;39:502-06

28. Heo DH, Jun AY, Cho YJ. Magnetic resonance neurography findings in herpetic brachial plexopathy. J Neurol 2011;258:137-39

29. Ryan M, Twair A, Nelson E, et al. Whole body magnetic resonance imaging in the diagnosis of Parsonage Turner syndrome. Acta Radiol 2004;45:534-39

30. Melis B, Defranco MJ, Lädermann A, et al. The teres minor muscle in rotator cuff tendon tears. Skeletal Radiol 2011;40:1335-44

31. Chhabra A, Lee P, Bizzell C, et al. High resolution 3-Tesla magnetic resonance neurography of musculocutaneous neuropathy. J Shoulder Elbow Surg 2012;21:e1-6

32. Tagliafico AS, Michaud J, Marchetti A, et al. US imaging of the musculocutaneous nerve. Skeletal Radiol 2011;40:609-16 